\title{
Differential Effects of Koisio Technology-Modulated Solutions on the Growth of Lung Fibroblast Cell Cultures and Lung Cancer Cell Cultures
}

Mingchao Zhang*, Yinghui Men*, Qi Zhu, Weihai Ying\#

Med-X Research Institute and School of Biomedical Engineering, Shanghai Jiao Tong University, Shanghai 200030, P.R. China

* These two authors contributed equally to this work.

\#: Corresponding author

Weihai Ying, Ph.D.

Professor, School of Biomedical Engineering and Med-X Research Institute

Shanghai Jiao Tong University

1954 Huashan Road

Shanghai, 200030, P.R. China

E-mail: weihaiy@sjtu.edu.cn

Running title: Koisio solutions modulate cell growth 


\begin{abstract}
Cell growth is a crucial biological property of cells, which plays key roles in several major biological processes including organ development, tissue repair and cancer development. It is of both scientific and medical significance to discover new strategies to modulate cell growth. Koisio technology is a novel technology that modulates the properties of water solely by physical approaches without additions of any external substances. In our current study we obtained the following findings regarding the effects of Koisio technology-modulated solutions on cell growth: First, compared with the lung fibroblast (L929) cell cultures cultured in normal media, the L929 cells cultured in Koisio technology-modulated media grew at approximately $20 \%$ higher speed; second, compared with the lung cancer cell cultures (LLC cells) cultured in normal media, the LLC cells cultured in Koisio technology-modulated media grew at approximately 9\% lower speed; and third, compared with the telomere lengths of the L929 cells cultured in normal media, the L929 cells cultured in Koisio technologymodulated media had approximately $14 \%$ longer telomere length. Collectively, our study has provided the first evidence indicating that Koisio technology-modulated solutions affect differentially the growth of lung fibroblast cell cultures and that of lung cancer cell cultures. The capacity of the Koisio technology-modulated solutions to promote the growth of lung fibroblast cell cultures may result at least partially from its capacity to protect the telomere length.
\end{abstract}

Keywords: Cell growth; fibroblasts; lung cancer cells; telomere; water 


\section{Introduction}

Cell growth is a critical biological property of cells, which profoundly affects multiple key biological processes such as organ development, tissue repair, and cancer development (1-6). Tissue repair can also be affected by numerous factors such as oxidative stress and $\mathrm{NAD}^{+}$metabolism (7-9). Under injury conditions, enhanced cell growth can lead to accelerated tissue repair (4). In contrast, aberrant and uncontrolled cell growth is a hallmark of cancer cells (5). Cell growth is controlled by multiple factors and highly complex mechanisms (6). Due to the profound significance of cell growth, it is critical to discover novel approaches to enhance normal cell growth and inhibit cancer cell growth.

It has been established that telomere shortening is a hallmark of cellular aging $(10,11)$. Prevention of telomere shortening is an important strategy for slowing the aging process $(10,11)$. It is of both scientific and biological significance to search for novel strategies to slow down telomere shortening.

Koisio technology is a novel technology that modulates the properties of water solely by physical approaches without additions of any external substances. A previous study has reported that the ceramics produced by Koisio technology could produce water with increased permeability through aquaporins (12). Our latest study has also found that Koisio technology-modulated media can lead to an approximately $57 \%$ increase in intracellular ATP levels. However, it has been unclear if Koisio technologymodulated solutions without additions of any external substances may affect cell growth. Due to the critical biomedical significance of cell growth, in current study we determined the effects of the solutions modulated by Koisio technology on cell growth of both lung fibroblast cell cultures and lung cancer cell cultures. Our study showed 
that the solutions modulated by Koisio technology produced differential effects on the cell growth of these two types of cells.

\section{Materials and Methods}

\section{Cell Cultures}

Mouse lung fibroblast (L929) cell lines $(13,14)$ were purchased from Chinese Academy of Sciences Cell Bank. Lung cancer (LLC) cell lines were purchased from American Type Culture Collection (CRL-1642 $\left.{ }^{\mathrm{TM}}\right)$. The cells were grown in DMEM (SH30243.01, Hyclone) supplemented with 10\% FBS (100-500, GEMINI), 100 U/mL penicillin, and $0.1 \mathrm{mg} / \mathrm{mL}$ streptomycin $\left(15140122\right.$, Gibco) at $37^{\circ} \mathrm{C}$ in humidified $5 \%$ $\mathrm{CO}_{2}$ atmosphere.

\section{Modulations of cell culture media by Koisio technology}

Modulations of cell culture media were conducted by Koisio technology as reported previously (12).

\section{CCK8 assay}

Cell viability was measured by using Cell Counting Kit-8 (Dojindo Laboratories, Kumamoto, Japan). In brief, cells were seeded in 96-well plates at the density of $5 \times 10^{3}$ per well. After $24 \mathrm{~h}, \mathrm{CCK} 8$ solution was added into the medium at a dilution of 1:10 
and incubated at $37^{\circ} \mathrm{C}$ for $1-3 \mathrm{~h}$. The absorbance at $450 \mathrm{~nm}$ was determined using a microplate reader (Synergy2, BioTek, Winooski, VT, USA).

\section{Determinations of telomere lengths by qPCR}

Cell's DNA was extracted by using DNA extraction kit (DP1902, Bioteke Inc, Beijing) according to the manufacturer's protocol. Mean telomere length was measured by Shanghai Biowing Applied Biotechnology CO. LTD, as described previously (15). qPCR was conducted in triplicate, and the reactions included $4 \mu$ of genomic DNA (80 ng), $\quad 0.1 \quad \mu 1$ of telomere primer $\quad(10 \mu \mu M) \quad$ (forwards: CGGTTTGTTGGGTTTGGGTTGGGTTTGGGTTTGGGTT; reverse: (GGCTTGCCTTACCCTTACCCTTACCCTTACCCTTACCCT), $0.1 \quad \mu 1$ of $\mathrm{YH}-1$ forwards primer $\quad(10 \quad \mu \mathrm{M}) \quad$ (5'-CGCACAGAGTAGTAAGGAAAGTGAAGTAGGCCGGGC-3'), $1 \mu \mathrm{l}$ of YH-1 reverse primer $(10 \mu \mathrm{M})\left(5^{\prime}-\right.$ GTGCTGGGATtACAGGCGTGAG-3'), $1 \mu \mathrm{l}$ of uniprimer2 (VICATGGACAGTGAGATCTGTCCAT-BHQ1CGCACAGAGTAGTAAG), and $10 \mu \mathrm{l}$ NovoStart ${ }^{\circledR}$ SYBR qPCR SuperMix, in a final reaction volume of $20 \mu l$.

For PCR product testing, we adopted universal molecular beacon technology and the telomere amplifications were detected using SYBR green dye. All PCRs were carried out on a 7500 Real-Time PCR System, and amplification was conducted as 
follows: Stage $1: 5 \mathrm{~min}$ at $95^{\circ} \mathrm{C}$, Stage $2: 30$ cycles (telomere and internal gene reaction) of $15 \mathrm{sec}$ at $95^{\circ} \mathrm{C}$, Stage $3: 1 \mathrm{~min}$ at $50^{\circ} \mathrm{C}$, Stage $4: 45 \mathrm{sec}$ at $72^{\circ} \mathrm{C}$, and fluorescence signals were collected at $50^{\circ} \mathrm{C}$. The reactions were carried out in a 96 -well plate. Software v2.3 was used for analysis. The telomere length for each sample was determined using the telomere to high-copy gene ratio $(\mathrm{T} / \mathrm{H}$ ratio) by calculating the $\triangle \mathrm{Ct}[\mathrm{Ct}($ telomere)/Ct (high-copy gene)]. The $\mathrm{T} / \mathrm{H}$ ratio for each sample (x) was normalized to the mean $\mathrm{T} / \mathrm{S}$ ratio (test sample/standard sample) of the reference sample $\left[2^{-(\triangle \mathrm{Ctx}-2 \triangle \mathrm{Ctr})}=2^{-\triangle \Delta \mathrm{Ct}}\right]$, which was also used for the standard curve as a reference sample and as a validation sample. In every run, two reference samples were included to validate each reaction. The experiment was considered reliable if the $\mathrm{T} / \mathrm{H}$ ratio of the control sample ranged within the 95\% variation interval (0.95-1.05).

\section{Statistical analyses}

All data are presented as mean \pm SEM. Data were analyzed by student $t$-test. $P$ values less than 0.05 were considered statistically significant.

\section{Results}

Lung fibroblast (L929) cell cultures cultured in Koisio technology-modulated DMEM media (KM) showed significantly faster growth speed compared with the L929 cells cultured in regular DMEM media 
We determined the effects of KM on the growth of L929 cells - a lung fibroblast cells. Compared with the L929 cells cultured in regular DMEM media, the L929 cells cultured in KM grew at approximately $20 \%$ higher speed (Fig. 1).

The lung cancer (LLC) cell cultures cultured in KM showed significantly slower growth speed compared with the lung cancer cell cultures cultured in regular DMEM media

We also determined the effects of KM on the growth of LLC cells - a lung cancer cell line. Compared with the LLC cells cultured in regular DMEM media, the LLC cells cultured in KM did not grow at faster speed, instead, the cells grew at approximately 9\% slower speed (Fig. 2).

L929 cells cultured in KM had significantly longer telomere lengths compared with the $\mathbf{L 9 2 9}$ cells cultured in regular DMEM media

Since telomere shortening is a hallmark of cellular aging, we determined the effects of KM on the telomere length of L929 cells. Compared with the L929 cells cultured in regular DMEM media, the L929 cell cultures cultured in KM had approximately $14 \%$ longer telomere length (Fig. 3).

\section{Discussion}

The major findings of our study include: First, compared with the lung fibroblast (L929 cells) cell cultures cultured in normal media, the lung fibroblast cell cultures 
cultured in KM grew at approximately $20 \%$ higher speed; second, compared with the lung cancer cell cultures (LLC cells) cultured in normal media, the LLC cells cultured in KM grew at approximately 9\% lower speed; and third, compared with the telomere lengths of the L929 cells cultured in normal media, the L929 cells cultured in KM had approximately $14 \%$ longer telomere length.

The growth rate of normal cells is of great biomedical value. A relatively high growth rate of normal cells can lead to higher speed of tissue repair if an organ or tissue is injured. Our study has indicated that the lung fibroblast cell cultures cultured in KM grew at approximately $20 \%$ higher speed, suggesting that Koisio technology-based solutions may enhance the growth rate of normal cells as well as the tissue repair speed.

Cancer cells are cells with aberrant and uncontrolled growth. Therefore, it is critical to determine if KM may also accelerate the growth rate of cancer cells. Our study has found that compared with the LLC cells cultured in normal cell culture media, the LLC cell cultures cultured in KM did not grow at a higher speed, in instead, the cells grew at approximately $9 \%$ slower speed.

Collectively, our study has suggested that Koisio technology-modulated media affect differentially the growth rate of normal lung fibroblast cell cultures and lung cancer cell cultures. However, the mechanisms underlying the differential effects of $\mathrm{KM}$ on the growth rate of normal lung cells and lung cancer cells remain unclear. It is warranted to conduct future studies to investigate the mechanisms underlying these differential effects.

Telemere shortening is a major index of in vitro aging of cells $(10,11)$. Prevention of telemere shortening is an important strategy for slowing down cellular aging $(10,11)$. Our study has shown that compared with the telomere lengths of the 
L929 cells cultured in normal media, the L929 cells cultured in KM had approximately $14 \%$ longer telomere length. This finding has suggested that KM may enhance the growth speed of L929 cells at least partially by slowing down the shortening of telemere length of the cells. 


\section{References:}

1. Mishra, P., and Chan, D. C. (2014) Mitochondrial dynamics and inheritance during cell division, development and disease. Nat Rev Mol Cell Biol 15, 634646

2. Lim, H. Y. G., and Plachta, N. (2021) Cytoskeletal control of early mammalian development. Nat Rev Mol Cell Biol 22, 548-562

3. Boroughs, L. K., and DeBerardinis, R. J. (2015) Metabolic pathways promoting cancer cell survival and growth. Nat Cell Biol 17, 351-359

4. Rodrigues, M., Kosaric, N., Bonham, C. A., and Gurtner, G. C. (2019) Wound Healing: A Cellular Perspective. Physiol Rev 99, 665-706

5. Hanahan, D., and Weinberg, R. A. (2011) Hallmarks of cancer: the next generation. Cell 144, 646-674

6. Texada, M. J., Koyama, T., and Rewitz, K. (2020) Regulation of Body Size and Growth Control. Genetics 216, 269-313

7. Ying, W., and Xiong, Z. G. Oxidative stress and NAD+ in ischemic brain injury: current advances and future perspectives. Curr Med Chem 17, 2152-2158

8. Ying, W., Wei, G., Wang, D., Wang, Q., Tang, X., Shi, J., Zhang, P., and Lu, H. (2007) Intranasal administration with $\mathrm{NAD}^{+}$profoundly decreases brain injury in a rat model of transient focal ischemia. Front Biosci 12, 2728-2734

9. Zhang, M., and Ying, W. (2019) $\mathrm{NAD}^{+}$Deficiency Is a Common Central Pathological Factor of a Number of Diseases and Aging: Mechanisms and Therapeutic Implications. Antioxid Redox Signal 30(6):890-905.

10. Turner, K. J., Vasu, V., and Griffin, D. K. (2019) Telomere Biology and Human Phenotype. Cells $\mathbf{8}$ 
11. Aubert, G., and Lansdorp, P. M. (2008) Telomeres and aging. Physiol Rev 88, $557-579$

12. Kozumi, T., and Kitagawa, Y. (2016) Water structure changes induced by ceramics can be detected by increased permeability through aquaporin. Biochem Biophys Rep 5, 353-358

13. Cheng, Y., Luo, W., Li, Z., Cao, M., Zhu, Z., Han, C., Dai, X., Zhang, W., Wang, J., Yao, H., and Chao, J. (2019) CircRNA-012091/PPP1R13B-mediated Lung Fibrotic Response in Silicosis via Endoplasmic Reticulum Stress and Autophagy. Am J Respir Cell Mol Biol 61, 380-391

14. Ma, J. W., Huang, B. S., Hsu, C. W., Peng, C. W., Cheng, M. L., Kao, J. Y., Way, T. D., Yin, H. C., and Wang, S. S. (2017) Efficacy and Safety Evaluation of a Chlorine Dioxide Solution. Int J Environ Res Public Health 14: 329.

15. Sun, G., Cao, H., Bai, Y., Wang, J., Zhou, Y., Li, K., and Xiao, J. H. (2021) A novel multiplex qPCR method for assessing the comparative lengths of telomeres. J Clin Lab Anal 35, e23929 


\section{Figure Legends:}

Fig.1. The lung fibroblast (L929 cells) cell cultures cultured in Koisio technologymodulated DMEM media (KM) showed significantly faster growth speed compared with the L929 cells cultured in regular DMEM cell culture media. Compared with the L929 cells cultured in normal cell culture media, the L929 cells cultured in KM grew at approximately $20 \%$ higher speed. From Passage 2, a part of the LLC cells grew in the KM. CK assay were conducted when the cell growth reached Passage 10 - 16. The results were collected from five independent CCK assays on five passages of cells. $\mathrm{N}=30, P<0.05$.

Fig.2 The lung cancer cell cultures (LLC cells) cultured in Koisio technologymodulated DMEM media (KM) showed slower growth speed compared with the LLC cells cultured in regular DMEM cell culture media. Compared with the LLC cells cultured in normal media, the LLC cells cultured in KM grew at approximately 9\% lower speed. From Passage 2, a part of the LLC cells grew in the KM. CCK assay were conducted when the cells reached Passage $10-16$. The results were collected from the five independent CCK assays on five passages of cells. $\mathrm{N}=30, P<0.05$.

Fig. 3. The lung fibroblast (L929 cells) cell cultures cultured in Koisio technologymodulated DMEM media (KM) had significantly longer telomere lengths compared with the L929 cells cultured in regular DMEM cell culture media. Compared with the telomere lengths of the L929 cells cultured in normal cell culture media, the L929 cells cultured in KM had approximately 15\% longer telomere length. From Passage 2, a part of the L929 cells grew in KM. Assays of telomere lengths of 
bioRxiv preprint doi: https://doi.org/10.1101/2022.03.02.482600; this version posted March 2, 2022. The copyright holder for this preprint (which was not certified by peer review) is the author/funder. All rights reserved. No reuse allowed without permission.

the cells were conducted when the cells reached Passage $17-19$. The results were collected from three independent assays on three passages of cells. $\mathrm{N}=9, P<0.01$. 


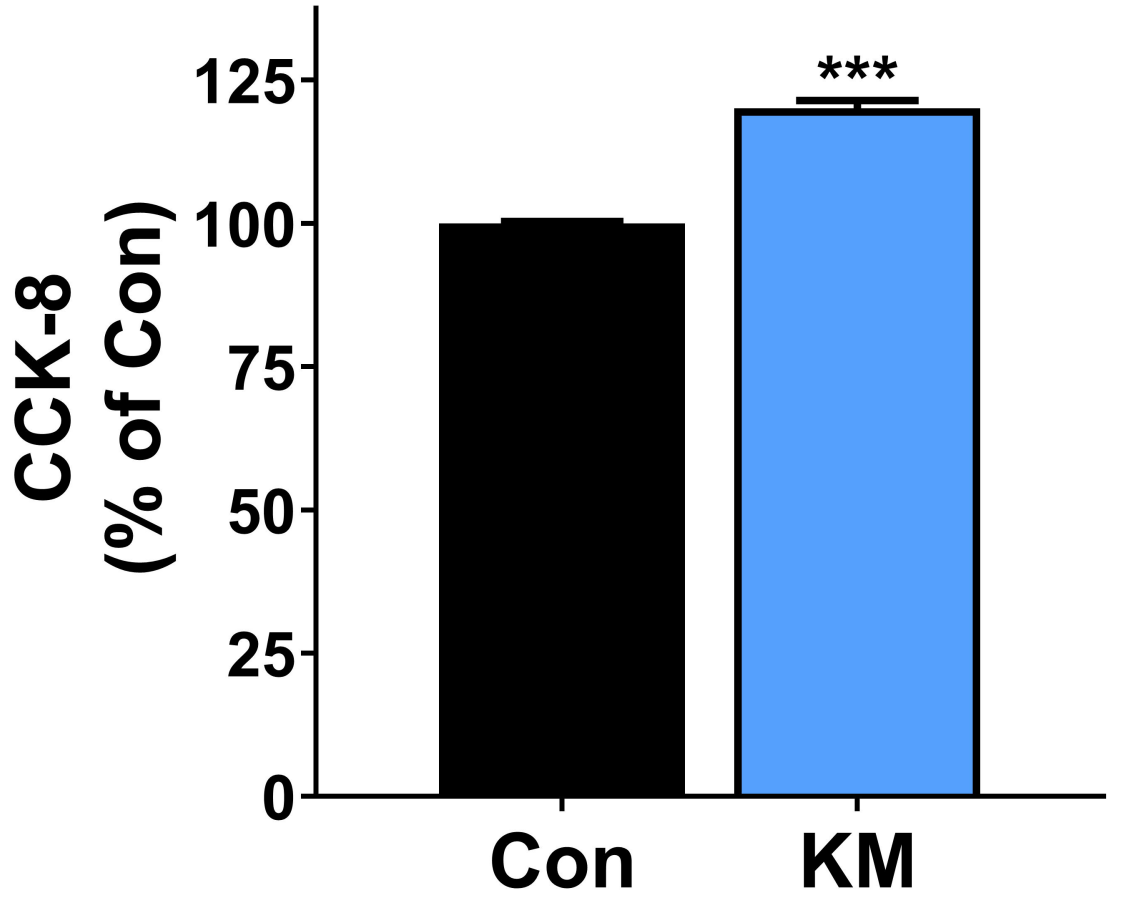




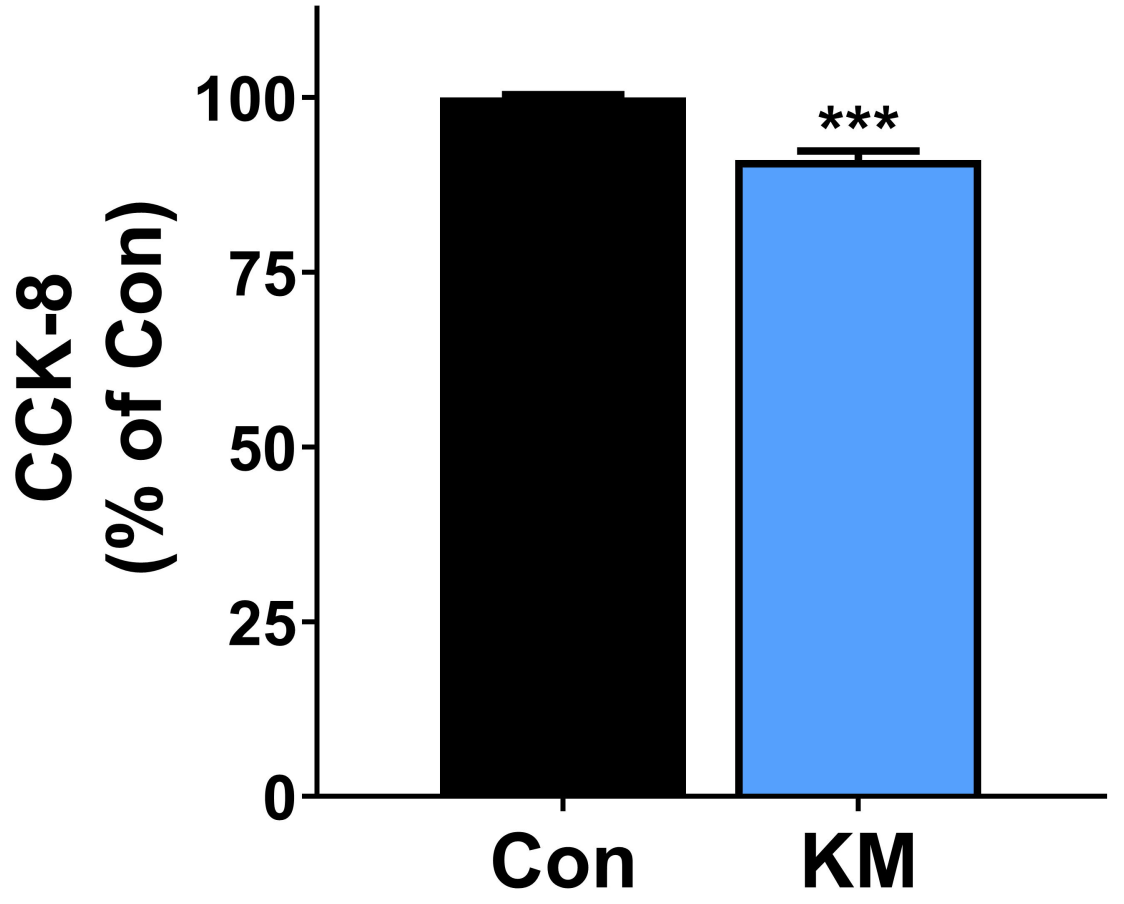




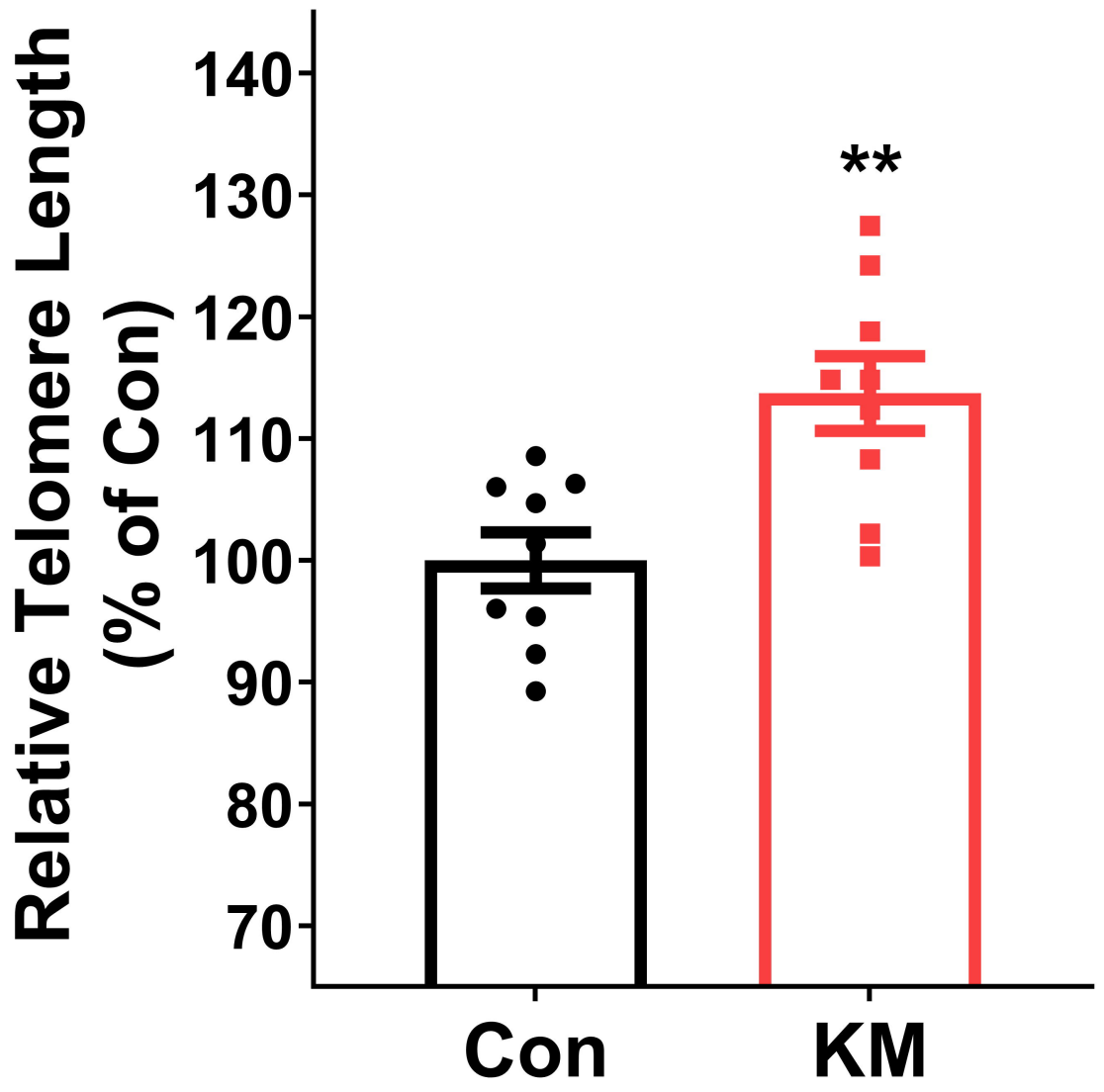

